\title{
Equilíbrio eletrolítico e ácido-base em bovinos
}

\author{
Eletroctrolyte and acid-base balance in bovines
}

\begin{abstract}
Moisés Dias Freitas ${ }^{I^{*}}$ Marina Guimarães Ferreira ${ }^{\mathrm{I}}$ Paulo Marcos Ferreira ${ }^{\mathrm{I}}$ Antônio Último Carvalho ${ }^{I}$ Andrey Pereira Lage ${ }^{I I}$ Marcos Bryan Heinemann ${ }^{I I}$ Elias Jorge Facury Filho ${ }^{\mathrm{I}}$
\end{abstract}

\section{- REVISÃO BIBLIOGRÁFICA -}

\section{RESUMO}

O conhecimento sobre a fisiologia e regulação dos eletrólitos e do equilíbrio ácido-base é necessário, pois a saúde do animal depende diretamente da composição normal dos fluídos nos diversos compartimentos corporais. Esta regulação está intimamente entrelaçada em um rigoroso e preciso controle, de modo que uma anormalidade em um destes compartimentos reflete na alteração dos demais. A avaliação do equilibrio eletrolítico e ácido-base pode fornecer informações fundamentais para o diagnóstico, prognóstico e decisão terapêutica de diversas enfermidades nos bovinos, tais como diarreias neonatais, hemoparasitoses (anaplasmose e a babesiose), deslocamento de abomaso e processos de sépticos. As correções destas alterações com medidas terapêuticas adequadas são pontos chave para minimizar as perdas por mortalidade causadas por estas enfermidades.

Palavras -chave: equilibrio ácido-base e equilibrio eletrolítico, bovinos.

\section{ABSTRACT}

The knowledge of the physiology and regulation of electrolytes and blood gas parameters is necessary because the health of the animal depends directly on the normal composition of fluid in body compartments. The regulation of these is closely intertwined with a rigorous and precise control so that an abnormality in one of these compartments reflects the change in the other. The evaluation of electrolyte and acidbase balance can provide key information for diagnosis, prognosis and therapeutic decision for several diseases in animals such as neonatal diarrhea, anaplasmosis and babesiosis, displacement of abomasum and septic processes. The corrections of these changes with appropriate treatment are key to minimizing losses due to mortality in these diseases.

Key words: acid-base balance, electrolyte balance, bovine.

\section{INTRODUÇÃO}

A manutenção da homeostase é diretamente dependente do equilíbrio dinâmico que há entre os líquidos corporais, $\mathrm{pH}$ e eletrólitos (CUNNINGHAM, 2004). Nos mamíferos, os líquidos e eletrólitos estão distribuídos em, basicamente, dois compartimentos: intracelular (LIC) e o extracelular (LEC). A dinâmica de distribuição dos líquidos e eletrólitos que compõem o organismo dos mamíferos, como a manutenção em termos de volume e composição, é essencial para processos metabólicos fundamentais à vida. Já o $\mathrm{pH}$ destes líquidos atua na manutenção estrutural e funcional de proteínas, enzimas e organelas celulares (CONSTABLE, 1999; KASARI, 1999; CUNNINGHAM, 2004).

$\mathrm{Na}$ medicina veterinária, todas as enfermidades que cursam com desidratação, (diarreias, hemoparasitoses, broncopneumonias, deslocamento de abomaso, endotoxemias, retenção dos anexos fetais, entre outros) causam paralelamente alterações no equilíbrio ácido-base e eletrolítico e, embora seja comum a ocorrência destas enfermidades nas propriedades rurais, o tratamento quase nunca é adequado, pois a administração de antibióticos e anti-inflamatórios não corrige os distúrbios hidroeletrolíticos e ácido-base,

'Departamento de Clínica e Cirurgia Veterinárias, Escola de Veterinária (EV), Universidade Federal de Minas Gerais (UFMG), Av. Antônio Carlos, 6627, 30123-970, Belo Horizonte, MG, Brasil. Email: m_diasfreitas@yahoo.com.br.*Autor para correspondência "Departamento de Medicina Veterinária Preventiva, EV, UFMG, Belo Horizonte, MG, Brasil 
que são as principais causas da alta taxa de mortalidade destas afecções.

O objetivo desta revisão de literatura é ressaltar a importância do equilíbrio ácido-base e eletrolítico para a manutenção da homeostase e consequente vida dos animais.

A água é componente presente no corpo dos mamíferos em maior quantidade. Em indivíduos adultos, a água corporal total, que é o volume hídrico deste, corresponde a aproximadamente $60 \%$ do peso corporal total, enquanto em animais jovens esse percentual aumenta, sendo em torno de $70 \%$. O percentual da água corporal total pode variar em decorrência de determinadas doenças (THORNTON \& ENGLISH, 1978; BERCHTOLD, 1999; DIBARTOLA, 2000).

O LIC é todo o conteúdo hídrico que está localizado no interior das células e representa $2 / 3$ da água corporal total, ou seja, cerca de $40 \%$ do peso corporal. Os demais $1 / 3$ da água corporal total, ou seja, os $20 \%$ restantes compõem o LEC, que está subdividido em outros três compartimentos: o plasma, o fluido intersticial e ainda o fluido transcelular (KIRBY \& RUDLOFF, 1997; DIBARTOLA, 2000; KOCHEVAR, 2003)

Os fluidos transcelulares ou líquidos compartimentalizados são produzidos por células especializadas e são representados pelo fluido cerebroespinhal, sinovial, bile, linfa, secreções respiratórias, dentre outros (BAER, 1996; DIBARTOLA, 2000).

O LIC e o LEC estão em equilíbrio dinâmico, embora difiram entre si quanto à composição eletrolítica e ao volume. Nestes, a água difunde-se livremente entre os meios, através das membranas celulares, sendo seu volume controlado pela força osmótica de cada compartimento corpóreo. Tanto nos animais saudáveis quanto nos doentes, os volumes do LIC e LEC são reflexos da partição dos fluidos corpóreos e solutos, portanto, a estimativa das alterações do equilíbrio hídrico e eletrolítico é importante tanto para a compreensão da fisiologia quanto da patogenia da afecção, bem como para administração do tratamento (fluidoterapia) (CARLSON, 1997; WALKER et al., 1998; BERCHTOLD, 1999; DIBARTOLA, 2000).

O equilíbrio osmótico entre o LIC e o LEC é mantido pelos eletrólitos, que são os solutos, e, para manter a homeostase do organismo, é necessário que haja a neutralidade entre os meios, ou seja, deve haver uma equivalência entre cátions e ânions (BAER, 1996; KELLUM, 2000; ROSE \& POST, 2001).

A osmolalidade refere-se à força osmótica que solutos exercem em soluções e está diretamente relacionada com o número de partículas em solução. A osmolalidade efetiva ou tonicidade refere-se à osmolalidade do meio, quando os solutos que a determinam não são livremente permeáveis. A osmolalidade é medida em miliosmoles de soluto por quilograma de solução (mOsm kg-1) (CARLSON, 1997; ROSE \& POST, 2001).

A osmolalidade plasmática (LEC) é diretamente dependente da concentração sérica dos eletrólitos, principalmente do sódio. Dessa forma, a avaliação do hiato osmolal é uma forma de avaliar a concentração de solutos osmoticamente ativos no sangue $\left(\mathrm{Na}^{+}, \mathrm{K}^{+}\right.$, glicose e nitrogênio ureico sanguíneo (BUN)) (MEYER et al., 1995; RADOSTITS et al., 2007; KANEKO et al., 2008). A osmolalidade é calculada a partir da seguinte fórmula (RADOSTITS et al., 2007):

Osmolalidade $=1,86([\mathrm{Na}+]+[\mathrm{K}+])+\frac{\text { Glicose }}{18}+\frac{\text { BUN }}{2,8}+8,6$

Os valores normais para a osmolalidade do plasma giram em torno de $300 \mathrm{mOsm} \mathrm{kg}^{-1}$ (CARLSON, 1997; RADOSTITS et al., 2007; KANEKO et al., 2008). Considerando-se que os fluidos corpóreos são isotônicos, em condições fisiológicas, a osmolalidade do LIC também pode ser estimada (BAER, 1996).

$\mathrm{O}$ sódio $\left(\mathrm{Na}^{+}\right)$é o principal cátion presente no $\mathrm{LEC}$, enquanto o cloreto $\left(\mathrm{Cl}^{-}\right)$e o bicarbonato $\left(\mathrm{HCO}_{3}{ }^{-}\right)$ são os principais ânions. Já no LIC o potássio $\left(\mathrm{K}^{+}\right)$é o cátion em maior abundância, além do cálcio $\left(\mathrm{Ca}^{+2}\right)$ e o magnésio $\left(\mathrm{Mg}^{+2}\right)$, enquanto os fosfatos e as proteínas são os principais ânions (CARLSON, 1997; DIBARTOLA, 2000). Existem outros eletrólitos compondo os líquidos corporais (Tabela 1).

O conhecimento da fisiologia da regulação do $\mathrm{pH}$ e dos fluidos corporais é de fundamental importância para a compreensão das alterações orgânicas e metabólicas em decorrência dos distúrbios hídricos, eletrolíticos e/ou de ácido-base em animais (CUNNINGHAM, 2004; HOUPT, 2006).

A regulação do LIC e do LEC envolve uma inter-relação hormonal (renina, aldosterona, hormônio antidiurético - $\mathrm{ADH}$, fator natridiurético - FNA), o monitoramento gerado por receptores no sistema circulatório e a composição eletrolítica (ANGELOS \& VAN MESTRE, 1999). O sódio é principal cátion do LEC, que contém cerca de $75 \%$ do sódio corporal total (CARLSON, 1997; CUNNINGHAM, 2004). O volume do LEC, assim como sua osmolalidade, é determinado pelo conteúdo do $\mathrm{Na}^{+}$e seus ânions acompanhantes. Desta forma, um aumento na concentração de $\mathrm{Na}^{+}$pode levar à expansão do LEC, com consequente hipertensão sistêmica e formação de edemas. Da mesma forma, a redução deste eletrólito resulta em hiponatremia, 
Tabela 1 - Valores de referência dos principais eletrólitos, janela aniônica, $\mathrm{HCO}_{3}{ }^{-}$, $\mathrm{pH}$ venoso e osmolalidade dos bovinos.

\begin{tabular}{|c|c|c|c|c|c|}
\hline \multirow[b]{2}{*}{ Parâmetros } & \multicolumn{5}{|c|}{ 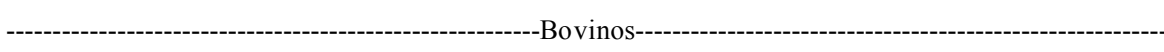 } \\
\hline & $\begin{array}{l}\text { DIRKSEN et } \\
\text { al., } 1993 \\
\text { Bezerros }\end{array}$ & $\begin{array}{l}\text { CARLSON, } \\
1997\end{array}$ & $\begin{array}{l}\text { RASDOSTITS } \\
\text { et al., } 2007\end{array}$ & $\begin{array}{l}\text { KANEKO et al., } \\
2008\end{array}$ & $\begin{array}{l}\text { FREITAS et al., } \\
2009 \text { bezerro } \\
\text { (3-30 dias) }\end{array}$ \\
\hline $\mathrm{Na}\left(\mathrm{mEq} \mathrm{L}^{-1}\right)$ & $115-145$ & $132-152$ & $132-152$ & $132-152$ & $136,41-137,65$ \\
\hline $\left.\mathrm{K}(\mathrm{mEq} \mathrm{L})^{-1}\right)$ & $3,5-5,0$ & $3,9-5,8$ & $3,9-5,8$ & $3,9-5,8$ & $4,36-4,58$ \\
\hline $\mathrm{Cl}\left(\mathrm{mEq} \mathrm{L}^{-1}\right)$ & $75-115$ & $97-111$ & $95-110$ & $97-111$ & $98,36-99,82$ \\
\hline $\mathrm{Ca}\left(\mathrm{mEq} \mathrm{L}^{-1}\right)$ & $1,8-2,5$ & $9,7-12,4$ & $9,7-12,4$ & $9,7-12,4$ & - \\
\hline $\operatorname{Mg}\left(\mathrm{mEq} \mathrm{L}^{-1}\right)$ & $0,8-1,2$ & $1,8-2,3$ & $1,8-2,3$ & $1,8-2,3$ & - \\
\hline $\mathrm{P}\left(\mathrm{mEq} \mathrm{L}^{-1}\right)$ & $2,0-3,5$ & $5,6-6,5$ & $5,6-6,5$ & $5,6-6,5$ & - \\
\hline Janela aniônica $\left(\mathrm{mEq} \mathrm{L}^{-1}\right)$ & - & $13,9-20,2$ & 17,2 & $10-20$ & $11,79-12,94$ \\
\hline $\mathrm{HCO}_{3}\left(\mathrm{mEq} \mathrm{L}^{-1}\right)$ & $23-29$ & $20-30$ & $20-30$ & $17-29$ & $29,55-30,60$ \\
\hline Osmolalidade $\left(\mathrm{mOsm} \mathrm{kg}^{-1}\right)$ & - & $270-300$ & $270-306$ & - & - \\
\hline $\mathrm{pH}$ & $7,33-7,37$ & $7,35-7,50$ & $7,35-7,50$ & $7,31-7,53$ & $7,399-7,420$ \\
\hline
\end{tabular}

ocasionando a redução do LEC e desidratação (DIBARTOLA, 2000; ROSE \& POST, 2001).

$\mathrm{O} \mathrm{Na}{ }^{+}$é essencial para o potencial de membrana, que é de fundamental importância para inúmeras funções celulares especializadas, como as contrações musculares, transmissão de impulsos nervosos, entre outros. A manutenção da sua concentração corporal é controlada unicamente pela ingestão e excreção (DIBARTOLA, 2000; CUNNINGHAM, 2004).

Absorção do $\mathrm{Na}^{+}$ocorre no intestino delgado em um processo passivo e está ligada ao movimento de solutos ativamente absorvidos ou secretados. Esta absorção ocorre em maior abundância com a presença de glicose e aminoácidos, pois estes são cotransportadores desse íon para o interior dos enterócitos na membrana em borda escova (DESJEUX et al., 1977; SMITH, 2009).

A distribuição do $\mathrm{Na}^{+}$nos líquidos corporais é bem diferente daquela prevista pelo potencial de membrana dos organismos vivos. Este fato é compensado pela ação da $\mathrm{Na}^{+}-\mathrm{K}^{+}$adenosinatrifosfatase ou simplesmente "bomba de $\mathrm{Na}^{+}-\mathrm{K}^{+}$- ATPase", que, com gasto de energia, retira o $\mathrm{Na}^{+}$e introduz o K${ }^{+}$no interior das células (CARLSON, 1997; ROSE \& POST, 2001).

As alterações na concentração de $\mathrm{Na}^{+}$ sérico, hipernatremia ou hiponatremia, são condições frequentes em animais enfermos. As afecções gastrointestinais que por ventura venham a causar diarreia em bovinos, por exemplo, estão comumente associadas a casos de hiponatremia, da mesma forma que perdas sanguíneas e insuficiência adrenal. Já a insuficiência cardíaca congestiva, a insuficiência hepática e a síndrome nefrótica são exemplos de afeç̧ões que causam hipernatremia (CARLSON, 1997; DIBARTOLA, 2000; RADOSTITS et al., 2007).

A concentração de $\mathrm{Na}^{+}$sérico não pode ser associada ao conteúdo de sódio total do organismo, uma vez que pacientes com hiponatremia ou hipernatremia podem apresentar redução, aumento ou ainda conteúdo de sódio corporal total normal (ANGELOS \& VAN MESTRE, 1999; DIBARTOLA, 2000). As consequências das alterações na concentração sérica do $\mathrm{Na}^{+}$estão na hiperosmolalidade para animais hipernatrêmicos e, normalmente, mas nem sempre, na hiposmolalidade para animais hiponatrêmicos (DIBARTOLA, 2000).

Da mesma forma que o sódio é determinante para o volume do LEC, o potássio é o maior responsável pela pressão osmótica intracelular. Aproximadamente 98\% do potássio disponível para o organismo encontra-se no LIC, sendo sua distribuição associada ao sódio, pela "bomba de $\mathrm{Na}^{+}-\mathrm{K}^{+}$- ATPase" (CARLSON, 1997).

$\mathrm{O} \mathrm{K}^{+}$está relacionado com diversas funções bioquímicas, transmissões dos impulsos nervosos, contrações musculares e também influência no metabolismo de carboidratos. Os requerimentos dietéticos de $\mathrm{K}^{+}$normalmente são atendidos, pois sua concentração é elevada nos alimentos, sendo necessária excreção deste íon para evitar a toxicidade. Nos bovinos e equinos a maior parte do $\mathrm{K}^{+}$é excretada pela urina, mas o suor, as secreções do trato gastrointestinal e as fezes também contribuem para a eliminação deste íon (CARLSON, 1997).

Os distúrbios na concentração de potássio alteram a relação entre o $\mathrm{K}^{+}$intra e extracelular e seu 
gradiente transmembrana, acarretando mudanças no potencial elétrico da membrana celular e gerando distúrbios na contração miocárdica e neuromuscular (CUNNINGHAM, 2004). O equilíbrio da concentração deste íon envolve a regulação extracorpórea, que está relacionada com o consumo dietético e as perdas orgânicas diárias, e a regulação interna, que está relacionada com o equilíbrio ácido-base, glicemia, insulinemia, exercício e a liberação de catecolaminas (CARLSON, 1997).

As afecções gastrointestinais, como vômitos e diarreia, contribuem para a redução dos estoques calêmicos corpóreos, embora haja uma hipercalemia. Este fato ocorre em decorrência da alteração do balanço interno do $\mathrm{K}^{+}$, a partir da redistribuição deste nos meios intra e extracelular (CARLSON, 1997; ROSE \& POST, 2001).

Acompanhante do sódio, o cloro é o ânion mais abundante no LEC, presente também em baixas concentrações no LIC. Está presente nos alimentos, comumente associado com o sódio ou potássio. A absorção, distribuição e excreção do $\mathrm{Cl}^{-}$ocorre de forma passiva, sendo distribuído de acordo com os gradientes elétricos originados pelo transporte ativo do $\mathrm{Na}^{+}$(CARLSON, 1997; DIBARTOLA, 2000; CUNNINGHAM, 2004). O cloro é um importante componente em muitas secreções, como suor, saliva e secreções gástricas (ROSSELL \& ROUSSEL, 2007).

As alterações da cloremia normalmente estão associadas às alterações da natremia e do balanço hídrico. A hipercloremia é normalmente observada em animais com acidose metabólica, sendo a alta concentração deste íon associada ao aumento da reabsorção renal do cloreto, em resposta à baixa relação entre os íons cloreto e bicarbonato, devido à redução do bicarbonato plasmático. A concentração sérica do cloro tende a variar inversamente à concentração de bicarbonato (DIBARTOLA, 2000; ROSE \& POST, 2001; CUNNINGHAM, 2004). As alterações na cloremia, quando não associadas à natremia e à hidratação, estão associadas a algum distúrbio ácido-base (ROSE \& POST, 2001; ROSSELL \& ROUSSEL, 2007).

Em bovinos, o deslocamento de abomaso leva a uma alcalose metabólica moderada com hipocalemia e hipocloremia, provavelmente devido à atonia abomasal, produção contínua de ácido clorídrico e obstrução parcial da saída de seu conteúdo, o que resulta em sequestro do cloro no abomaso e refluxo deste para o rúmen. A hipocalemia resulta, provavelmente, da diminuição de ingestão de alimentos e da contínua secreção renal destes eletrólitos (CONSTABLE et al., 1991).

As mudanças nas concentrações séricas dos íons devem ser interpretadas juntamente com as alterações na concentração total de $\mathrm{CO}_{2}\left(\mathrm{TCO}_{2}\right)$ e pH, pois a dinâmica dos íons no LEC e LIC é modificada nos caso de acidose ou alcalose metabólica. Um exemplo desta alteração está no movimento do potássio que sai do LIC para o LEC, quando há acidose metabólica e esta não está associada ao aumento da concentração sérica de ácidos orgânicos (acetona e lactato) (ADROGUE \& MADIAS, 1981; ROSSELL \& ROUSSEL, 2007).

Dessa forma, tanto o equilíbrio eletrolítico quanto o equilíbrio ácido-base estão entrelaçados e ambos são fatores essenciais para a mantença da homeostase no organismo de bovinos. A manutenção do pH em valores fisiológicos é de extrema importância uma vez que inúmeros processos bioquímicos são influenciados por enzimas intracelulares, as quais têm sua atividade excelente somente em determinada faixa de variação de pH (CUNINNGHAM, 2004; HOUPT, 2006).

$\mathrm{O} \mathrm{pH}$ do sangue venoso dos bovinos varia de 7,31 a 7,41, sendo os valores inferiores a esta variação indícios de acidose e, para valores superiores, alcalose (CARLSON, 1997; RADOSTITS et al., 2007, KANEKO et al., 2008).

A regulação do pH está entrelaçada com um rigoroso e preciso controle da concentração de íons $\mathrm{H}^{+}$intra e extracelulares. Este processo é realizado, basicamente, por três vias metabólicas que estão interligadas. São elas: tampões intracelulares e extracelulares, eliminação de $\mathrm{CO}_{2}$ via pulmonar e regulação renal de íons $\mathrm{H}^{+}$e $\mathrm{HCO}_{3}{ }^{-}$(DRAGE \& WILKINSON, 2001; ROSE \& POST, 2001).

Os tampões presentes no LIC e no LEC são constituídos de pares conjugados de ácidos e bases fracas, sendo estas substâncias capazes de doar ou receber prótons respectivamente, além disso, de se dissociarem parcialmente, tornando a regulação do $\mathrm{pH}$ mais eficiente (DIBARTOLA, 2000). Os tampões intracelulares são as proteínas, fosfatos orgânicos e inorgânicos e a hemoglobina, nos glóbulos vermelhos. Já no meio extracelular, o sistema bicarbonato - ácido carbônico é, basicamente, o responsável pelo tamponamento (CARLSON, 1997).

A troca catiônica realizada pelas células é outro recurso do organismo que pode auxiliar o tamponamento dos meios intra e extracelular. Este processo é realizado pela membrana celular, constituindo-se no movimento de íons $\mathrm{H}^{+}$através da membrana na troca por $\mathrm{K}^{+}$e Na ${ }^{+}$(ROSE \& POST, 2001).

O sistema de tamponamento intracelular é realizado principalmente por proteínas, que constituem o tampão mais numeroso do organismo. Estas proteínas podem exercer tanto o papel de ácidos quanto de bases 
fracas, dependendo de seu arranjo espacial (CARLSON, 1997). A hemoglobina é a proteína que atua como tampão intracelular mais abundante no organismo, cerca de $80 \%$, e tem a capacidade de fixar $\mathrm{CO}_{2} \mathrm{e} \mathrm{H}^{+}$. A albumina é outro tampão importante no meio extracelular e tem a capacidade de ligar-se com íons $\mathrm{H}^{+}$(CARLSON, 1997; ROSE \& POST, 2001).

Já no meio extracelular, o par conjugado bicarbonato - ácido carbônico é o sistema de tamponamento mais importante. O bicarbonato é abundante no LEC e pode ser recuperado pelos rins, enquanto o $\mathrm{CO}_{2}$, produzido pela respiração celular tecidual, pode ser eliminado pela ventilação alveolar nos pulmões, caracterizando um sistema aberto (ROSE \& POST, 2001).

$\mathrm{O} \mathrm{CO}_{2}$ produzido nos tecidos, ao difundirse na corrente sanguínea, combina-se instantaneamente com $\mathrm{H}_{2} \mathrm{O}$, formando o ácido carbônico $\left(\mathrm{H}_{2} \mathrm{CO}_{3}\right)$, um ácido fraco, que, devido a sua instabilidade, dissocia-se em $\mathrm{H}^{+}{\mathrm{e} \mathrm{HCO}_{3}}^{-}$. Esta reação é mediada pela anidrase carbônica, que está presente nos eritrócitos (DIBARTOLA, 2000): $\mathrm{H}^{+}+\mathrm{HCO}_{3}{ }^{-}$ $\mathrm{H} 2 \mathrm{CO}_{3}<-\mathrm{H}_{2} \mathrm{O}+\mathrm{CO}_{2}$.

Nos eritrócitos, os íons $\mathrm{H}+$ se ligam, na maior parte, à hemoglobina. Dessa forma, os íons bicarbonato saem das células, na troca com íons cloretos, em um mecanismo conhecido como desvio de cloreto. Já no meio extracelular, o bicarbonato irá atuar como tampão. Esta via transporta boa parte do $\mathrm{CO}_{2}$ produzido pela respiração sem alterar o pH (DIBARTOLA, 2000).

O sistema bicarbonato - ácido carbônico é o sistema de tamponamento mais importante. A concentração sérica do bicarbonato é, em média, de $24 \mathrm{mEq} \mathrm{L}^{-1}$, entretanto, a concentração do ácido carbônico não pode ser mensurada devido a sua alta instabilidade (CUNNINGHAM, 2004; HOUPT, 2006). Há uma relação direta entre a concentração de $\mathrm{CO}_{2}$ no sangue, determinada pela pressão arterial parcial de $\mathrm{CO}_{2}, \mathrm{PACO}_{2}$, e a quantidade de ácido carbônico $\left(\mathrm{H}_{2} \mathrm{CO}_{3}\right)$ sanguínea. Desta forma, a determinação da $\mathrm{PACO}_{2}$ é o método utilizado para a avaliação da concentração do $\mathrm{H}_{2} \mathrm{CO}_{3}$. O valor do $\mathrm{PACO}_{2}$ é de $40 \mathrm{mmHg}$, e a constante de $\mathrm{CO}_{2}$ no sangue é de $0,03 \times \mathrm{PACO}_{2}(\mathrm{CARLSON}, 1997)$. A $\mathrm{PACO}_{2}$ e a concentração sérica do íon bicarbonato formam o par de tampões utilizados para o monitoramento do $\mathrm{pH}$ no LEC (ROSE \& POST, 2001).

Além do tamponamento intra e extracelular, o sistema respiratório também exerce grande influência no mecanismo de controle do $\mathrm{pH}$, uma vez que o $\mathrm{CO}_{2}$ é o responsável pela maior parte da carga ácida produzida durante os processos metabólicos (DRAGE \& WILKINSON, 2001). Os pulmões respondem rapidamente às modificações de $\mathrm{pH}$, alterando a taxa de remoção de $\mathrm{CO}_{2}$ do sangue e, consequentemente, a taxa de $\mathrm{H}_{2} \mathrm{CO}_{3}$ (CUNNINGHAM, 2004; HOUPT, 2006).

Os pulmões agem na oxigenação sanguínea e na ventilação alveolar. A pressão arterial de oxigênio $\left(\mathrm{PAO}_{2}\right)$ é o parâmetro utilizado para a avaliação da oxigenação pulmonar, enquanto a $\mathrm{PACO}_{2}$ é utilizada

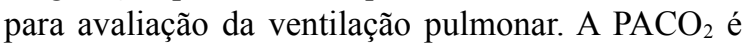
inversamente proporcional à função alveolar. Tal fato implica que pequenas mudanças na ventilação pulmonar alteram rapidamente os teores de $\mathrm{CO}_{2}$ e de íons $\mathrm{H}^{+}$, bem como os valores do $\mathrm{pH}$ (GOMES, 1997).

A terceira linha de defesa do equilíbrio ácido-base envolve o sistema renal. Os rins são responsáveis pela composição do LEC e pela reabsorção e regeneração dos íons bicarbonato presentes no ultrafiltrado glomerular, sendo este processo associado à excreção de íons $\mathrm{H}^{+}$ (CUNNINGHAM, 2004).

Os rins agem na eliminação dos excessos de ácidos e bases, de origem não respiratória, portanto, somente a função renal é incapaz de restabelecer o equilíbrio ácido-base. Os ácidos fixos excretados na urina reagem com os tampões presentes nesta. A ação dos tampões urinários, principalmente os fosfatos e a amônia, evitam a queda brusca do $\mathrm{pH}$ urinário (ROSE \& POST, 2001; CUNNINGHAM, 2004).

A hemogasometria é uma excelente ferramenta para a avaliação do estado ácido-base e prognóstico do quadro clínico. Estes parâmetros são importantes, uma vez que a interpretação das alterações dos sinais clínicos, em decorrência dos distúrbios hídricos, eletrolíticos e entre ácidos e bases, é imprecisa e está diretamente relacionada com o risco de morte dos enfermos (GOMES, 1997).

Os valores de $\mathrm{pH}$ fora da faixa de normalidade indicam acidose ou alcalose. Entretanto, valores de $\mathrm{pH}$ aparentemente normais não descartam a possibilidade de alterações no equilíbrio ácido-base (CARLSON, 1997).

Os valores de $\mathrm{pH}$ estão diretamente associados às atividades metabólicas e à função respiratória. Portanto, alterações nos valores normais da $\mathrm{PACO}_{2}$ são indícios de distúrbios no equilíbrio ácidobase. Os valores fisiológicos da $\mathrm{PACO}_{2}$ para os bovinos variam de 34,7 a 44mmHg (CARLSON, 1997; GOMES, 1997). O aumento de $\mathrm{PACO}_{2}$ é chamado de acidose respiratória primária e ocorre normalmente nas afecções respiratórias. A redução dos valores de $\mathrm{PACO}_{2}$ são indicativos de alcalose respiratória, comuns em hiperventilação (geralmente associada à resposta à acidose metabólica) ou em decorrência de distúrbios neurológicos (MEYER et al., 1995; GOMES, 1997; DIBARTOLA, 2000). 
O bicarbonato pode ser mensurado como padrão e sérico. $\mathrm{O} \mathrm{HCO}_{3}{ }^{-}$padrão se refere aos valores plasmáticos, sem levar em consideração as influências respiratórias, ou seja, este valor é calculado considerando que a $\mathrm{PACO}_{2}$ é de $40 \mathrm{mmHg}$. Já o bicarbonato sérico é aquele medido diretamente no sangue (BOOKALLIL, 2009).

Os valores fisiológicos de $\mathrm{HCO}_{3}$ p plasmático para bovinos giram em torno de $27 \mathrm{mmol} \mathrm{L}^{-1}$. O bicarbonato é o alcalinizante mais importante do equilíbrio ácido-base e está diretamente relacionado à capacidade de tamponamento do sangue. As causas mais frequentes de redução de $\mathrm{HCO}_{3}$ em bovinos', levando a uma acidose metabólica, são as diarreias, a cetose e a acidose lática ruminal. Já nas alcaloses metabólicas primárias, relacionadas ao aumento da concentração de $\mathrm{HCO}_{3}{ }^{-}$, os vômitos e o deslocamento de abomaso nos bovinos são as principais causas (MEYER et al., 1995; CARLSON, 1997; CUNNINGHAM, 2004).

O dióxido de carbono total $\left(\mathrm{TCO}_{2}\right)$ é a soma dos valores de bicarbonato (maior parte do $\mathrm{CO}_{2}$ ) e $\mathrm{CO}_{2}$ dissolvido, dando uma estimativa grosseira do estado ácido-base do paciente. Os valores fisiológicos, nos bovinos, do $\mathrm{TCO}_{2}$, variam de 25,6 a $33,4 \mathrm{mEq} \mathrm{L}^{-1}$. Valores reduzidos de $\mathrm{TCO}_{2}$ são indicativos de acidose e aumentados de alcalose. Como os valores de $\mathrm{TCO}_{2}$ são diretamente dependentes das influências metabólicas e respiratórias, a interpretação de seus resultados é limitada (MEYER et al., 1995; CARLSON, 1997).

O valor do excesso de base (EB) é definido como a quantidade de ácido, em $\mathrm{mEq} \mathrm{L}^{-1}$, necessário para restabelecer o $\mathrm{pH}$ de um litro de sangue, a $37^{\circ} \mathrm{Ce}$ $\mathrm{PACO}_{2}$ de 40mmHg ao valor de 7,4 (BOOKALLIL, 2009) ou, simplesmente, a diferença entre o total de bases e ácidos tituláveis (CARLSON, 1997). O excesso de base, desvio de base ou a diferença de base é um subsídio para a identificação de distúrbios metabólicos (GOMES, 1997). Os valores negativos indicam uma deficiência de bases, caracterizando um quadro de acidose clínica devido à redução de $\mathrm{HCO}_{3}^{-}$, já os casos de valores positivos sugerem alcalose metabólica (CARLSON, 1997).

Os valores de EB também são utilizados para avaliar o estado ácido-base. A vantagem de se utilizar este parâmetro é que este valor permanece praticamente constante durante as alterações agudas da $\mathrm{PACO}_{2}$, refletindo apenas alterações metabólicas, sofrendo pouca ou nenhuma interferência das alterações respiratórias (MEYER et al., 1995; TIETZ et al., 1996; CARLSON, 1997).

A janela aniônica, ou ânion gap, é a diferença entre cátions e ânions medidos no sangue.
Esta diferença é virtual, pois o princípio básico da homeostase é a manutenção da neutralidade elétrica. Como não são mensurados todos os eletrólitos presentes no sangue, para simplificação da definição, adota-se como valor de janela aniônica a seguinte equação (CONSTABLE, 1999): Janela aniônica $=$ $\left[\mathrm{Na}^{+}+\mathrm{K}^{+}\right]-\left[\mathrm{Cl}^{-}+\mathrm{HCO}_{3}^{-}\right]$.

Estes íons são utilizados, para este cálculo, por estarem em maior concentração e importância no LEC. As cargas dos cátions não medidos no sangue tendem a balancear as cargas dos ânions não medidos. Os cátions não medidos são o cálcio e o magnésio, enquanto os ânions são as proteínas, o fosfato e os sulfatos. Os valores normais da janela iônica, para a maioria das espécies, estão entre $10-20 \mathrm{mEq} \mathrm{L}^{-1}$ ou 10 20 $\mathrm{mmol} \mathrm{L}^{-1}$ (CARLSON, 1997).

Nos bovinos, os valores normais da janela aniônica variam de $12-18 \mathrm{mmol} \mathrm{L}^{-1}$. O aumento destes valores sugere uma acidose metabólica ou insuficiência renal (retenção de ácidos). A avaliação deste parâmetro é outra ferramenta utilizada para a determinação do prognóstico clínico do enfermo (TIETZ et al., 1996; MEYER et al., 1995; DIBARTOLA, 2000).

Todos os desequilíbrios primários entre ácidos e bases geram respostas do organismo na tentativa de corrigir o $\mathrm{pH}$ e retornar aos parâmetros fisiológicos. Entretanto, com exceção dos quadros de acidose respiratória crônica, nenhuma resposta compensatória é capaz de restabelecer o $\mathrm{pH}$ a seu valores fisiológicos (GOMES, 1997). A tabela 2 relaciona as respostas compensatórias das alterações primárias.

\section{CONCLUSÃO}

Inúmeras são as enfermidades, nos bovinos, capazes de comprometer a homeostase do organismo, podendo gerar desordens no equilíbrio eletrolítico e ácido-base. As possíveis consequências para o organismo, em decorrência dessas alterações, variam desde inibição de mecanismos celulares, vasoconstricção cerebral e até morte dos enfermos.

Portanto o conhecimento da fisiologia dos mecanismos de regulação do equilíbrio eletrolítico e ácido-base é de extrema importância para a determinação do prognóstico clínico e para a escolha do tratamento adequado, minimizando, dessa forma, as possíveis perdas econômicas por diminuição da produção ou até mesmo pela morte dos animais.

As pesquisas com equilíbrio ácido-base nas enfermidades de bovinos no Brasil são escassas. Esse fato é, em parte, responsável pela pouca utilização da fluidoterapia nos animais. Assim, faz-se necessária a realização de trabalhos de pesquisa que criem 


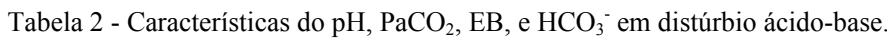

\begin{tabular}{ccccc}
\hline $\mathrm{pH}$ & $\mathrm{PaCO}_{2}$ & $\mathrm{HCO}_{3}^{-}$ & $\mathrm{EB}$ & \multicolumn{1}{c}{ Alteração } \\
7,4 & $40 \mathrm{mmHg}$ & $24 \mathrm{mEq} \mathrm{L}^{-1}$ & 0 & Normalidade \\
\hline$\uparrow$ & $\downarrow$ & $\downarrow$ & 0 ou & Alcalose respiratória \\
$\uparrow$ & $\uparrow$ & $\uparrow$ & + & Alcalose metabólica \\
$\downarrow$ & $\uparrow$ & $\uparrow$ & 0 & Acidose respiratória \\
$\downarrow$ & $\downarrow$ & $\downarrow$ & - & Acidose metabólica \\
$\downarrow$ & $\uparrow$ & $\uparrow$ & - & Acidose mista respiratória e metabólica \\
$\uparrow$ & $\downarrow$ & $\uparrow$ & + & Alcalose mista respiratória e metabólica \\
7,4 & $\uparrow$ & $\downarrow$ & - & Distúrbio misto acidose alcalose \\
7,4 & $\downarrow$ & $\downarrow$ & Distúrbio misto alcalose acidose \\
\hline
\end{tabular}

Fonte: Adaptado de BOOKALLIL (2009)

propostas de correção ácido-base e hidroeletrolítica nos bovinos enfermos, empregáveis no campo, garantindo maior eficiência nos tratamentos e diminuindo a mortalidade.

Os custos com equipamentos e insumos para realização de hemogasometria são altos, portanto, isso ainda limita seu uso no campo. Há disponibilidade de tecnologia para realização desses exames a campo, ainda onerosos, porém com perspectivas de se tornarem mais econômicos.

\section{AGRADECIMENTO}

Ao Conselho Nacional de Desenvolvimento Científico e Tecnológico (CNPq) e a Fundação de Amparo a Pesquisa do Estado de Minas Gerais (FAPEMIG) pelo suporte financeiro.

\section{REFERÊNCIAS}

ADROGUE, H.J.; MADIAS, N.E. Change in plasma potassium concentration during acute acid-base disturbances. American Journal of Medicine, v.71, p.456-467, 1981

ANGELOS, S.M.; VAN MESTRE, D.C. Treatment of sodium balance disorders: water intoxication and salt toxicity. Veterinary Clinics of North America: Food Animal Practice, v.15, n.3, p.587-606, 1999

BAER, R.W. Body fluid compartments. In: GUYTON, A.C.; HALL, J.E. Textbook of medical physiology. Philadelphia: Saunders, 1996. Cap.25, p.297-308.

BOOKALLIL, M.J. pH of the blood: acid base balance, 2009. Disponível em: <http://www.anaes.med.usyd.edu.au/ lectures/acidbase_mjb/acidbase.html.> Acesso em: 20 set. 2009.

CARLSON, G.P. Fluid electrolyte and acid-base balance. In: KANECO, J. Clinical biochemistry of domestic animals. 5.ed. London: Academic, 1997. p.485-515.

CONSTABLE, P.D. et al. Preoperative prognostic indicators on cattle with abomasal volvulus. Journal of the American Veterinary Medical Association, v.198, p.2077-2085, 1991.
CONSTABLE, P.D. Clinical Assessment of acid-base status: strong ion difference theory. Veterinary Clinics of North America: Food Animal Practice, v.15, n.3, p.447-472, 1999.

CUNNINGHAM, J.G. Tratado de fisiologia veterinária. 3.ed. Rio de Janeiro: Guanabara Koogan, 2004. 454p.

DiBARTOLA, S.P. Fluid therapy in small animal practice. 2.ed. Philadelphia: Saunders, 2000. 720p.

DIRKSEN, G. et al. Exame clínico dos bovinos. 3.ed. Rio de Janeiro: Guanabara Koogan, 1993. 419p.

DESJEUX, J.F. et al. Effects of sugars and amino acids on sodium movement across small intestine. American Journal of Diseases of Children, v.131, p.331-340, 1977.

DRAGE, S.; WILKINSON, D. Acid-base balance. Pharmacology, v.3, n.12, p.1-13, 2001.

GOMES, O.M. Interpretação clínicas das alterações ácidobásicas e distúrbio da oxigenação. Belo Horizonte: Edictor, 1997. 254p.

FREITAS, M.D. Avaliação dos parâmetros clínicos e de patologia clínica em bezerros naturalmente infectados com diarréia neonatal. 2009. 81f. Dissertação (Mestrado em Medicina Veterinária) - Escola de Veterinária, Universidade Federal de Minas Gerais, MG.

HOUPT, T.R.. Equilibrio ácido-básico. In: SWENSON, M.J.; REECE, W.O.O. Dukes fisiologia dos animais domésticos. 12.ed. Rio de Janeiro: Guanabara Koogan, 2006. Cap.9, p.147160.

KANEKO, J.J. et al. Clinical biochemistry of domestic animal. 6.ed. San Diego, California, USA Elsevier, 2008. $918 \mathrm{p}$.

KASARI, T.R. Metabolic acidosis in calves. Veterinary Clinics of North America: Food Animal Practice, v.15, n.3, p.473-482, 1999.

KELLUM, J.A. Determinants of blood $\mathrm{pH}$ in health disease. Critical Care, v.4, n.1, p.6-14, 2000. 
KIRBY, R.; RUDLOFF, E. The critical need for colloids: maintaining fluid balance. Compendium on Continum Education for the Practicing Veterinarian, v.19, p.705717, 1997.

KOCHEVAR, D.T. Princípios de equilíbrio ácido - básico: terapia hídrica e eletrolítica. In: ADAMS, H.R. Farmacologia e terapêutica em veterinária. 8.ed. Rio de Janeiro: Guanabara Koogan, 2003. Cap.25, p.417-444

MEYER, D.J. et al. Medicina de laboratório veterinária Interpretação e diagnóstico. São Paulo: Rocca, 1995. 308p.

RADOSTITS, O.M. et al. Veterinary Medice: a textbook of the diseases of cattle, horses, sheep, pigs and goats. 10.ed. Philadelphia: Elsevier, 2007. 2156p.

ROSE, D.B.; POST, T.W. Clinical physiology of acid base and electrolyte disorders. 5ed. New York: McgrawHill, 2001. 992p
ROSSEL, K.E.; ROUSSEL, A.J. Evaluation of the ruminant serum chemistry profile. Veterinary Clinics of North America: Food Animal Practice, v.23, n.3, p. 406-426, 2007.

SMITH, B.P. Large animal internal medicine. 4.ed. Missouri: Mosby Elsevier, 2009. 380-390p.

TIETZ, N.W. et al. Blood Gases and pH. In: BURTIS, Tietz textbook of clinical chemistry. 4.d. Philadelphia: Saunders, 1996. 506-522p.

THORNTON J.R.; ENGLISH, P.B. Body water of calves; changes in distribution with diarrrhoea. British Veterinary Journal, v.134, p.445-453, 1978.

WALKER, P. et al. A reliable, practical, and economical protocol for inducing diarrhea and severe dehydration in the neonatal calf. Canadian Journal of Veterinary Research, v.62, n.3, p.205-213, 1998 . 